\title{
A DETAILED MODEL OF THE DISTRIBUTION OF SYNCHROTRON EMISSION IN THE GALACTIC DISK
}

\author{
A. BROADBENT ${ }^{1}$, C.G.T. HASLAM ${ }^{2}$, and J.L. OSBORNE ${ }^{1}$ \\ ${ }^{1}$ Department of Physics, University of Durham, South Road, \\ Durham DH1 $1 A H$, England \\ ${ }^{2}$ Max-Planck-Institute for Radioastronomy, Auf dem Hügel 69, \\ D-5s00 Bonn 1, FRG
}

\begin{abstract}
A technique for separating the radio continuum emission of the Galaxy into its thermal and nonthermal components has been recently developed by Broadbent, Haslam and Osborne (1989). In this the thermal component is identified by its detailed correlation with the $60 \mu \mathrm{m}$ infrared emission as observed by IRAS after the subtraction of zodiacal light and the HI-associated dust emission. This technique has been applied to the $408 \mathrm{MHz}$ allsky survey of Haslam et al. (1982). A model of the distribution of synchrotron emissivity in the galactic disk has then been derived including information on the other tracers of spiral structure (HI, CO and giant HII regions) in order to account in detail for the observed nonthermal emission. The spiral arm pattern has two pairs of arms emanating from a central ellipse. The function describing the underlying variation of synchrotron emissivity with galactocentric radius is zero at the centre, rises to a sharp peak and then falls off slowly beyond $3 \mathrm{kpc}$. Using a scale length of the variation of cosmic ray electron density derived from $\gamma$-ray observations, we find that the scale length of the magnetic field variation must be as long as $22 \mathrm{kpc}$. This agrees with the scale length derived assuming equipartition between energy densities of magnetic field and cosmic rays. The variation of emissivity with height above the plane deduced by Phillipps et al. (1981) when included in our model gives good fits to the observed cuts across the plane. We have modelled the variation of the galactic magnetic field across a spiral arm as a gaussian. In order to fit the peaks in the galactic plane profile $\sigma=0.2 \mathrm{kpc}$ and a maximum compression of the field in the arm of 3.5:1 is required. These parameters are compatible with the results of $\mathrm{N}$-body simulations of spiral arm formation which treat gas clouds as the unit particles. A detailed description of the model is about to be submitted for publication.
\end{abstract}

\section{References}

Broadbent, A., Haslam, C.G.T. and Osborne, J.L., 1989. Mon. Not. R. astr. Soc., 237, 381.

Haslam, C.G.T., Salter, C.J., Stoffel, H. and Wilson, W.E., $1982 . \quad$ Astr. Astrophys. Suppl.,20, 37.

Phillipps, S., Kearsey, S., Osborne, J.L., Haslam, C.G.T and Stoffel, H., 1981. Astr. Astrophys.,103, 405. 